\title{
Are there really interactive processes in speech perception?
}

\author{
James M. McQueen ${ }^{1}$, Dennis Norris ${ }^{2}$ and Anne Cutler ${ }^{1}$ \\ ${ }^{1}$ Max Planck Institute for Psycholinguistics, Postbus 310, 6500 AH Nijmegen, The Netherlands \\ ${ }^{2}$ MRC Cognition and Brain Sciences Unit, 15 Chaucer Road, Cambridge CB2 2EF, UK
}

On both empirical and theoretical grounds, we argue that the affirmative answer of McClelland et al. [1] is premature. Contrary to the predictions of the TRACE model, which postulates interactive processing in speech perception, there is no lexically mediated compensation for coarticulation when there is a lexical bias in interpretation of the preceding fricative [2]. This cannot be dismissed with criticism of the fricative-final lexical contexts and their distance from the stops (Box 3 in Ref. [1]). The contexts in Ref. [2] were effective - there was a lexical bias on the fricatives; and the timing was appropriate - there was a compensation effect with the same fricative-stop gap in the nonword-context conditions. Furthermore, we suggest that the evidence on compensation that purports to show interaction is unconvincing. Some apparent lexical effects [3,4] are possibly higher-order transitional-probability effects [2]. Others perhaps reflect learning about experimentinternal biases; indeed, the effect in Ref. [5] did not seem to increase over time, but this null result is not definitive because learning could have occurred in the practice block.

Perceptual retuning can explain the selective-adaptation data (e.g. Ref. [6]). Retuning of phonetic perception can arise after only ten critical trials [7]; selective adaptation effects depend on many more exposures (e.g. 768 in Ref. [6]). Thus, selective adaptation can follow from perceptual retuning. After listeners learn that the ambiguous phoneme is $/ \mathrm{s} /$, for example, it acts as an adaptor, reducing the number of $/ \mathrm{s} /$ responses to the test stimuli. Further analysis of the data in Ref. [6] reveals exactly this: early blocks of trials show retuning, whereas later blocks show adaptation (Figure 3 in Ref. [8]).

The evidence on whether there is on-line lexical influence on prelexical processes is thus inconclusive. However, consensus has been reached on the existence of lexical feedback for learning $[7,9]$ and, as just shown, this can also explain apparent evidence of on-line interaction. Why might there be feedback that affects learning but not processing? Feedback for learning is helpful because it enables the listener to adjust to speaker-related variability [9], but feedback in on-line processing is not beneficial and could even be harmful [10]. These views are based on rational analysis [11]. Analyzing the nature of the perceptual task generated a hypothesis (that was confirmed in Ref. [9]) about how speech perception should operate.

According to the rational-analysis perspective, and McClelland et al. [1], the goal of the speech-recognition device is optimal interpretation. Bayesian methods

Corresponding author: McQueen, J.M. (james.mcqueen@mpi.nl)

Available online 25 October 2006. provide the optimal way to combine independent sources of information for perceptual decisions. However, if interaction were permitted between information sources, those sources would no longer be independent and the decision would be suboptimal [10]. Interaction, therefore, makes optimal interpretation harder. If an interactive algorithm could be made to compute the correct Bayesian decision function, the interaction would be a property only of that algorithm, not of the underlying computation. What would be computed is exactly what a non-interactive system would compute. Hence, commitment to the computational principle of optimality requires no commitment to the algorithmic principle of interactive processing.

No data require direct influences of the lexicon on prelexical mechanisms, and evidence and computational principles argue against interactive processing. Further evidence of lexical mediation of prelexical processes might yet appear. Indeed, if lexical retuning were implemented in the TRACE model using Hebbian learning [9,12], there could well be on-line processing effects. Such demonstrations would be further evidence of feedback in learning and not evidence of interactive processing, which, other than potentially as part of a learning mechanism, serves no useful function.

\section{References}

1 McClelland, J.L. et al. (2006) Are there interactive processes in speech perception? Trends Cogn. Sci. 10, 363-369

2 Pitt, M.A. and McQueen, J.M. (1998) Is compensation for coarticulation mediated by the lexicon? J. Mem. Lang. 39, 347-370

3 Elman, J.L. and McClelland, J.L. (1988) Cognitive penetration of the mechanisms of perception: compensation for coarticulation of lexically restored phonemes. J. Mem. Lang. 27, 143-165

4 Samuel, A.G. and Pitt, M.A. (2003) Lexical activation (and other factors) can mediate compensation for coarticulation. J. Mem. Lang. 48, 416-434

5 Magnuson, J.S. et al. (2003) Lexical effects on compensation for coarticulation: the ghost of Christmash past. Cogn. Sci. 27, 285-298

6 Samuel, A.G. (2001) Knowing a word affects the fundamental perception of the sounds within it. Psychol. Sci. 12, 348-351

7 Kraljic, T. and Samuel, A.G. Perceptual adjustments to multiple speakers. J. Mem. Lang. (in press)

8 Vroomen, J. et al. Visual recalibration and selective adaptation in auditory-visual speech perception: contrasting build-up courses. Neuropsychologia (in press)

9 Norris, D. et al. (2003) Perceptual learning in speech. Cogn. Psychol. 47, 204-238

10 Norris, D. et al. (2000) Merging information in speech recognition: feedback is never necessary. Behav. Brain Sci. 23, 299-370

11 Anderson, J.R. (1990) The Adaptive Character of Thought. Erlbaum

12 Mirman, D. et al. An interactive Hebbian account of lexically guided retuning of speech perception. Psychon. Bull. Rev. (in press) 